\title{
New Zealand's midwifery profession: Embracing graduate midwives' transition to practice
}

\author{
Mary Kensington ${ }^{A, B}$ MPH,BA, DipTchg (Tertiary), ADM, RM, RGON• Norma CampbellC MNZM, MA \\ (Mid), RM, RGON • Elaine Gray ${ }^{C} M$ Mid (Dist), Dip ALT, RM, RN • Lesley Dixon ${ }^{C}$ PhD, M Mid, BA \\ (Hons), RM - Emma Tumilty D, E PhD (cand.), PG Dip Heal Sci • Sally Pairman ${ }^{E}$ MNZM, D.Mid, MA, \\ $B M, R M, R G O N \cdot S u s a n$ CalvertF PhD (cand.), M Phil (Hons), B.Com, RM - Sue Lennox ${ }^{G}$ PhD, MA \\ (Applied), BA, RM, RN.
}

${ }^{\mathrm{A}}$ Corresponding

Author: mary.

kensington@cpit.

$\underline{\text { ac.nz }}$

${ }^{\text {B }}$ Christchurch

Polytechnic Institute of Technology

${ }^{\mathrm{C}}$ New Zealand

College of Midwives

${ }^{D}$ University of

Otago, New Zealand

${ }^{\text {E }}$ Otago Polytechnic, New Zealand

F Midwifery Council of New Zealand

${ }^{\mathrm{G}}$ Sky Blue House Limited, Wellington

\begin{abstract}
:
Background: The first year following registration is considered a stressful time for new health professionals as they transition from student to health practitioner, acclimatise to a new job and work environment, and become confident in their clinical skills. The Midwifery First Year of Practice (MFYP) programme was designed to provide support to graduate midwives during this transition.

Aim: This research explored the midwifery graduate's perspective of the MFYP programme to identify which elements were important and supported them in their transition to confident practitioner.

Method: Survey methodology was used to explore the views of the MFYP programme participants for the years 2007 to 2010 . This paper provides the qualitative analysis of the open text responses in the survey and explores the support needs of graduate midwives during their first year of practice.

Findings: A total of 180 midwives responded to the survey, with the majority also providing text responses to enable more in-depth understanding of their views. Graduate midwife participants were supported to consolidate practice skills and decision making, and to develop networks within the midwifery and wider health communities. An unexpected finding was that this support was provided by the whole midwifery community for all graduates, whether they were employed or self-employed. The mentoring relationship was also considered an important source of support.

Conclusion: Graduate midwives are gaining wrap-around support from the whole midwifery community. This research, unlike any previous international or national research, demonstrates that midwives in New Zealand have taken on collective responsibility for supporting graduates. This suggests that this national programme has successfully fostered a culture of nurturing midwife graduates into their professional role.
\end{abstract}

Keywords: Graduate midwife, mentor, transition, support, nurture, collective responsibility

\section{INTRODUCTION}

The realities of everyday practice can often be challenging for new health professionals as they transition from student to health practitioner. Newly registered health professionals need to adapt to the requirements of their new role and develop confidence. Confidence is a key theme identified by newly qualified midwives, who report that they often experienced a lack of confidence in their own abilities to make decisions based on their clinical assessments (Hobbs \& Green, 2003; Skirton et al., 2012). This lack of confidence more frequently stems from a lack of experience rather than a lack of competence or knowledge. In an Irish study of newly qualified midwives, Van der Putten (2008) found that the midwives often struggled to adapt to their new role. These midwives explained that the increased responsibility and awareness of accountability, often led to feelings of fear and insecurity. Similarly, an Australian study of newly qualified midwives, (Davis, Foureur, Clements, Brodie, \& Herbison, 2011) found that selfreported confidence to be able to practise, within the International Confederation of Midwives' definition and scope of practice of a midwife, was low for Australian graduates. The study reports this confidence increased only modestly within the first year of practice (Davis et al., 2011).

There is a statutory expectation that newly qualified midwives are able to provide midwifery care competently as soon as they have graduated. However, working on their own responsibility as a registered midwife requires additional support (Hobbs, 2012), and positive reinforcement for most practitioners (Skirton et al., 2012). The introduction of transition programmes has been an international response to the heightened anxiety and stress experienced by many newly registered health professionals. Transition programmes are structured to bridge the gap between being a student and being a practitioner. The programmes are designed to increase levels of confidence, consolidate knowledge and experience, and support critical reflection.

International research into various health professions has identified several essential elements of transition programmes (Altier \& Krsek, 2006; Banks et al., 2011; Bolden, Cuevas, Raia, Meredith, \& Prince, 2011; Cubit \& Ryan, 2011; Goode, Lynn, 
Krsek, \& Bednash, 2009; Herdrich \& Lindsay, 2006; Hillman \& Foster, 2011; Hobbs, 2012; Newhouse, Hoffman, Suflita, \& Hairston, 2007; O'Malley Floyd, Kretschmann, \& Young, 2005; Solowiej, Upton, \& Upton, 2010). These include: orientation to the environment, fostering a supportive working environment by providing a mentor or preceptor, continued clinical knowledge building and education, financial support, reflection and acknowledgement of learning at the end of the programme. Many transition programmes are industry-based and in health they are generally attached to the hospital. New Zealand has a unique model of maternity care and the midwife may be community or hospital based. Therefore, the New Zealand programme is uniquely designed to accommodate the midwife's choice of work place and the model of maternity care.

Midwives in New Zealand have a choice of work setting and can work within the community providing continuity of care to a caseload of women (Lead Maternity Carer (LMC) midwives) or within a maternity hospital setting (where they are known as core midwives). The challenge for the midwifery profession has been to develop and provide a transition programme that meets the individual needs of graduate midwives within the context of maternity care in New Zealand. The MFYP programme was developed for the New Zealand context by an expert advisory group as a strategy to provide support and enhance recruitment and retention for graduate midwives. Previously an informal ad hoc mentoring arrangement was in place, based around the New Zealand College of Midwives' consensus statement on mentoring (New Zealand College of Midwives, 2000).

\section{The challenge for the midwifery profession has been to develop and provide a transition programme that meets the individual needs of graduate midwives within the context of maternity care in New Zealand.}

The New Zealand College of Midwives commenced the MFYP programme in 2007 , the components of which include: facilitated support and orientation in clinical practice whatever the practice setting, one-to-one formal mentoring from a mentor chosen by the graduate midwife, funding to support the midwife to attend continuing professional development education, and supported participation in the profession's quality assurance process (Midwifery Standards Review). Each element of the programme is flexible and meets the needs of the graduate, wherever they work. There was a need to explore whether the programme contributed to both retention of graduates (Dixon et al., 2015) and which elements contributed to the development of professional confidence. The aim of this research was to explore the graduate midwife's perspective on participation in the MFYP programme and to identify which elements were important and supported them most in their transition to become a confident practitioner. Previous papers have provided evidence that the MFYP programme supports the retention of graduates in practice and is highly valued as a transition programme by participants (Dixon et al., 2015; Dixon et al., 2014; Pairman et al., 2015).

This paper has qualitatively analysed the survey's open text responses and explored the support needs of the graduate midwives and how these were met in practice during participation in the MFYP programme.

\section{METHOD}

Survey methodology was used to explore the views of the 415 participants of the MFYP programme between the years 2007 to 2010 inclusive. All participants were invited to complete a selfadministered survey which involved a range of questions about each aspect of the programme and provided open text comment boxes so that responses could be contextualised and explained more fully. A full description of the research methods has been previously published (Pairman et al., 2015).

An iterative thematic approach was used, whereby the qualitative survey data in the form of free text were explored for recurrent themes. The text responses were read closely by three members of the team (NC, MK, and SL) and themes were identified. Final themes were discussed and agreed. All the participants' survey responses were numbered to maintain anonymity throughout the analysis. All the quotes have been attributed to this number to maintain the participants' confidentiality. Ethics approval was provided by the Health and Disability Ethics Committees Upper South A Regional Ethics Committee. Ethics Reference: URA/12/ $\mathrm{EXP} / 012$.

\section{FINDINGS}

A total of 180 MFYP programme participants responded to the questionnaire and provided feedback on the programme. This gives a survey response rate of $43.4 \%$ of the 415 new graduates who completed the programme between 2007 and 2010. There was a large volume of text comments to each question providing context to the question response. The graduate midwives were asked where they worked in their first year of practice. The practice setting was either core/hospital or community/LMC work, with equal proportions of graduates practising in hospital as were practising in the community. They explained their choice of work setting was often determined by a need to consolidate practice (Pairman et al., 2015).

\section{I wanted hospital setting to ground me and increase my knowledge and confidence of abnormal childbirth/ pregnancy. So I had a sound foundation for all practice and settings. (Participant 047)}

\section{It was important to me to be able to develop and consolidate my practice as a LMC midwife and I felt strongly that this could only be done in the community. (Participant 034)}

Graduates from both settings identified the importance of their choice of setting as a means of consolidating and building their skills.

\section{Who provided practice support?}

Regardless of where a midwife works she will have specific support needs. The text data were analysed to determine how support was provided to meet the needs of the graduate midwives. Three themes were identified in regard to involvement of the midwifery community. These were:

- Support from midwifery colleagues

- Reassurance and encouragement

- The whole midwifery profession as a resource

Overall, graduates identified widespread support from their whole midwifery community. This included support in decision making, assessment and planning of care in practice. 


\section{Support from Midwifery Colleagues}

The graduates received support from a variety of midwifery colleagues within their midwifery community. This included the midwives they worked alongside, whether they were hospital midwives, LMC midwives, their mentors or the hospital midwifery managers.

The staff midwives who I worked with provided most of the clinical support I needed as they were there every day. My mentor was great if I need to talk about any issues. (Participant 077)

I had an amazing supportive group. We backed each other up at births until we didn't feel we needed it any more. We also had great support from core staff and management. My mentor was also available to talk when I needed her too. (Participant 160)

I worked with a variety of midwives and for the first year these were almost always very experienced. I was able to ask questions and discuss events with them as soon as this was appropriate. They were always available if I was unsure of something or wanted to find out why things were done a certain way (as I came to realise that all hospitals practised quite differently in terms of protocols, etc.). (Participant 031)

Clinical practice support was consistent, whether they were practising as an LMC midwife or as a hospital midwife.

\section{Reassurance and Encouragement}

Graduates described the reassurance, encouragement and support they gained from their collegial network within the profession and their practice environment. Feedback to the graduate was generally provided in a positive way with reassurance that they were meeting expectations for skills and knowledge.

Support involved reassurance I knew more than I gave myself credit for. Praising me when I had achieved things above the level that was expected or volunteered for what was more than expected for a graduate. Giving me chance to do something and help me plan, clarify why I would do something. (Participant 047)

I can't prioritise any one of these over another - they all provided the support that I needed according to the situation. Core midwives - experienced view of complex situations, available immediately at birthing units. Practice colleagues - support at home births or when I wanted a view from someone whose practice is philosophically aligned with my own. Practice partner - another new graduate. We attended births together in the first half of the year. (Participant 083)

The graduates indicated that feedback was individualised and dependent on the work setting. These comments emphasised that the whole profession, wherever and however they worked, was taking a role in reassuring and encouraging the graduates.

\section{The whole profession as a resource}

The midwifery profession as a whole appeared to be a valuable resource that was important for the graduate midwife. There were many comments which identified the value and importance of being able to discuss assessments and care planning with midwifery colleagues as well as receive feedback about the assessments/plans for particular situations.

Help with running ideas of next best step or option in the birthing process. My clinical skills were sound, it was just management of births that were no longer straight forward. I could run what I was thinking by other midwives at the hospital, Core midwives or other LMC's or my mentor, most were willing to help. (Participant 035)

It would appear that the graduates were able to gain support and discuss the care they provided, with a wide range of midwifery colleagues. There was overt willingness within the midwifery profession to help and support the graduate midwives. The graduates were also open to using this wide network of experience and readily available support as a resource for any issues, concerns or problems that occurred.

Another theme identified in the text data related to different types of support the graduate received from her mentor and this theme is analysed through the graduate/mentor relationship, mentor support and the mentor meetings.

\section{Graduate and mentor relationship}

The graduates described the difference between the clinical practice support they received from other colleagues and the support of their mentor. The mentor was most commonly seen as the person who helped the new graduate to consider and review her practice across the whole scope.

Core midwife for clinical support- mentor for emotional support and strategy to improve practise. (Participant 051)

I received support from my mentor in several ways. 1 . On the ground physical presence when needed. 2. Encouragement and confidence boosts. 3. Practical advice in clinical situations. 4. Advice with referrals, results, paperwork etc. (Participant 097)

Mentors supported graduates in many ways but it was evident their key role was particularly to enable reflection for the graduates on the wider clinical picture. Mentors also played a significant role in assisting the graduate in setting goals, debriefing and identifying areas for further learning.

\section{Trust and having a shared philosophy of practice appeared to be important elements which underpinned a positive mentoring relationship.}

The mentoring relationship was considered important, with the majority of graduate midwives $(n=148,82 \%)$ indicating that it was very important or important that they were able to choose their mentor.

She had to be someone that I completely trusted to maintain confidentiality; someone to whom I could reveal all my doubts and insecurities safely, and also someone who's clinical philosophies aligned with my own, and carried a demographically similar caseload. (Participant 014)

The ability to communicate well and relate to each other played a helpful role in my early mentorship - you may not get this with an assigned mentor. Also TRUST was a key factor as new grads are so vulnerable. (Participant 128)

There are times when we have to sit in a vulnerable position, it helped that I knew my mentor well during the most vulnerable times - the beginning weeks/months of practice. (Participant 080) 
Trust and having a shared philosophy of practice appeared to be important elements which underpinned a positive mentoring relationship. Graduates recognised their vulnerability and wanted to choose a mentor with whom they could safely reveal their insecurities and uncertainties.

There was a small proportion of graduate midwives (13.4\%) who responded that choosing a mentor was only somewhat important for them (Dixon et al., 2014). These midwives reflected on their decision in their text responses and suggested that perhaps their choice of mentor had not been optimal.

\begin{abstract}
On reflection having known what I know now I would have chosen a mentor a little more carefully. The mentor I would have liked was already mentoring someone else and my mentor offered to mentor me. As I didn't have any other ideas I accepted. (Participant 122)
\end{abstract}

A list of questions would be good to ask prospective mentors, so it is a good fit for the new grad midwife, so she is not just choosing someone she has worked with or gets on with on that basis only. (Participant 043)

These comments illustrate that while freedom to choose their own mentor is important, knowing how to choose a mentor is also important.

\section{Mentor support}

The respondents were asked how supportive they found the mentoring relationship to be. The majority $(n=156,86 \%)$ reported that they found the relationship very supportive or supportive with $18(10 \%)$ midwives reporting they felt unsupported or only somewhat supported.

\begin{abstract}
She was very straight up and told me what I needed to hear. There was no making things look rosy if they weren't. (Participant 023)
\end{abstract}

My mentor was always available, positive, empathetic, practical in her advice, and knowledgeable on practice issues. (Participant 143)

The mentor was seen as a trusted person chosen by the graduate and with whom she regularly met and developed a relationship. The mentor's role was seen as helping the individual graduate to consider and review her practice across the whole scope.
I felt supported by my mentor and was able to de-brief with her over the clinical side of things in a way that didn't always get to caught up in the actual doing of specific things... it was more of a 'helicopter' view, which allowed me to have a better understanding. (Participant 094)

\section{Mentoring Meetings}

Within the MFYP programme the mentor and the graduate are required to meet regularly or as identified by the graduate midwife. These meetings were outside of the clinical environment, at a convenient time and place, as negotiated between the two parties. The majority of graduates $(n=155,86.1 \%)$ found these meetings very helpful or quite helpful and explained that regular reviewing with a trusted colleague helped develop their reflective skills and deepened their understanding of practice.

Developed my skills in reflection and was a safe environment
to discuss care planning. (Participant 067)

I have never been good with critical reflection, however my mentor was very good at challenging me to do this. (Participant 122)
However, a small number of midwives $(n=11,6.1 \%)$ reported that they did not find the meetings helpful or only slightly helpful. Further analysis revealed that when the relationship was problematic the meetings were also problematic.

\section{I tried hard to please my mentor rather than honestly meeting my own needs. (Participant 081)}

It appears that the mentor relationship is instrumental in ensuring the graduate midwives feel supported and have their needs met.

For the majority of graduate midwives there was great satisfaction with the MFYP programme but there were a few who felt unsupported and who identified some dissatisfaction. This dissatisfaction appeared to be due to either culture or systems issues in their regions.

\section{Lack of support}

Where lack of support was a feature for 31 of the participants' first year experience, the reasons given were contextual such as: hospital staff shortages, negative attitudes of individuals or a hostile hospital culture. Of the 31 graduates, there were nine who commented they did not feel supported and a further 22 who suggested that there were some issues with support.

\section{The only times I felt unsupported were when it was so busy there was no one to ask. (Participant 101)}

\section{Staff shortages}

Lack of support was identified when the maternity facility was either understaffed or exceptionally busy.

\begin{abstract}
Sometimes the unit was understaffed and therefore the clinical support was stretched and unable to provide me with the support I needed - but this is the nature of the workplace setting. (Participant 80)
\end{abstract}

The only times I felt unsupported were when it was so busy there was no one to ask. (Participant 101)

It seemed that this lack of support was mostly transitory and short lived, although frequency was not measured by the survey.

\section{Negative maternity culture}

Eight graduates reported that they did not feel supported because of a culture within the maternity unit that did not consider new graduates should be working as LMC midwives.
I felt initially the core staff had an attitude of (and actually said this to me) 'if you think you're good enough to go straight out into independent practice you're good enough to not have any assistance.' (Participant 113)

Although this occurred for only a small number of the graduate respondents, the impact for them appeared to be significant.

\section{Summary of Findings}

This thematic analysis has identified that the graduates found each element of the programme important and contributed to building confidence. One of the key findings was the importance of support from the mentor and the wider midwifery community. This consistent support from their midwifery colleagues was identified by the graduates as pivotal in their transition experience. Each element of the programme was valued and made a significant contribution to the success of the transition to practice for these graduates in their first year. 
It is clear from these findings that it is the whole midwifery community who are providing support to New Zealand graduates. This professional network includes hospital midwives, LMC midwives, practice colleagues, mentors, midwifery managers, educators and others.

Midwifery relationships with women are a key element of professional practice for all midwives. For the graduate midwives, building relationships with colleagues and peers is also a vital part of their professional practice and necessary for successful transition to confident practitioner (Fenwick et al., 2012; Lennox, Jutel \& Foureur, 2012; Sullivan, Lock, \& Homer, 2011). A mixed methods study involving four New Zealand graduates found that there were few difficulties communicating with women; however, communicating with other health care professionals was found to be challenging. These challenges involved knowing how to ask for help appropriately, what information to give, and to whom and how to be assertive when necessary (Lennox, Jutel \& Foureur, 2012). The midwives in our study identified that they received support, reassurance and encouragement from the midwives around them.

\section{It is clear from these findings that it is the whole midwifery community who are providing support to New Zealand graduates. This professional network includes hospital midwives, LMC midwives, practice colleagues, mentors, midwifery managers, educators and others.}

Additionally, their midwifery colleagues were identified by the graduates as a resource with whom they could consult about practice decisions. This network of support and collegiality is a significant finding of this study and one we consider demonstrates that the midwifery partnership model, that is the basis for midwifery practice in New Zealand, is being reflected in the wider relationships that the graduates build within their midwifery community. The principles of the partnership model, which include: individual negotiation, informed choice and consent, equality, shared responsibility and empowerment, are identifiable in the comments and feedback from these graduate midwives (Guilliland \& Pairman, 2010).

Supportive relationships are an important means of socialisation into the culture of the group and can aid learning and skill acquisition (Mason \& Davies, 2013; Sullivan et al., 2011). The graduate midwives accessed support from their colleagues and the whole maternity community which was provided in a cooperative and collaborative way. Interaction with colleagues and a sense of belonging are associated with improved job satisfaction and are an important motivation to continuing to work as a midwife (Sullivan et al., 2011).

This sense of collective responsibility for supporting new graduates is evidence of a whole culture change within midwifery, which appears to have occurred within the past decade. This was not a theme seen in previous New Zealand research (Kensington, 2006; Lennox \& Foureur, 2012); neither is it found in other international studies of graduate midwives. Kensington (2006) in her study exploring the experiences of graduate midwives found that the graduates felt they were being 'tested' and 'on trial' in the hospital environment and often the mentor took on a 'protective role' at this time. This shift in culture within our profession is a new and exciting finding, demonstrating a positive change from the culture of 'eating our young' (Calvert, 2001; Hastie, 1995; Holland, 2001) to one that predominantly supports and nurtures graduate midwives as they take up their professional role.

\section{This sense of collective}

responsibility for supporting new
graduates is evidence of a whole
culture change within midwifery,
which appears to have occurred
within the past decade.

The increasing acceptance and support of graduates is an unanticipated positive consequence of the MFYP programme. It may have occurred due to the nationwide existence and consistency of the programme, along with the inbuilt education that supports the programme. Annual workshops are held that focus on the mentoring role, and have resulted in high attendance with more midwives attending than the number of those who have agreed to be mentors. This may have contributed to improved understanding of mentoring and increased knowledge of the mentoring role. Another potential explanation is that the very existence of the MFYP programme has given midwives the confidence to provide support to graduates knowing that there was a formal structure in process running alongside their input.

The mentoring relationship continues to be an important source of support for graduate midwives. The New Zealand College of Midwives' mentoring consensus statement describes the mentoring relationship as one of a negotiated partnership with the purpose of enabling and developing professional confidence (New Zealand College of Midwives, 2000). An important factor in building a positive relationship between the mentor and graduate is the freedom for graduates to choose their own midwife mentor. This appears to foster a relationship built on trust and respect. If the relationship is less than optimal then it can lead to less satisfaction with the overall programme. In a study of newly graduated United Kingdom midwives, Mason and Davies (2013) found that, despite evidence of bullying, good relationships between the newly qualified midwife and her preceptors were important and provided reciprocal benefits. The unique feature of the MFYP programme is its flexibility for the individual graduate whilst also providing a formal framework.

New Zealand graduate midwives are autonomous practitioners and this programme supports and enhances professional autonomy. It does this by enabling choice, with the graduates able to choose their mentor, work setting, learning goals and to identify the education workshops and professional development that best meet their individual needs. The programme also appears to support the development of supportive partnership relationships with their colleagues and mentors.

\section{STRENGTHS AND WEAKNESSES}

This study has provided insight into the perspectives of the graduate midwives who participated in the programme between 2007 and 2010. The qualitative themes provide more depth and context to previously reported data; however, they only represent the views of those who were invited and agreed to participate in the survey. There is the potential for response bias. 


\section{CONCLUSION}

The graduate midwives have identified that they receive support from the whole midwifery community which includes hospital midwives, LMC midwives, practice colleagues, mentors, midwifery managers, educators and others. The midwifery community was seen as a resource and provided reassurance and encouragement in a collegial and collaborative way. The MFYP programme supports autonomy with graduates enabled to have a range of choices. These choices includes where the graduate works, the mentor and the funded education they can access to support their individual professional development. Graduate midwives are encouraged to develop strong relationships with their colleagues that sit alongside the partnership relationship being established between the graduate midwife and the women she cares for. There appears to be a sense of collective responsibility taken by New Zealand midwives for supporting graduate midwives. This theme has not been uncovered in previous research nor is it seen in the international literature. It suggests the midwifery profession in New Zealand has moved to largely embrace a culture of providing professional support for its graduate midwives.

\section{ACKNOWLEDGEMENTS \& CONFLICT OF INTEREST DISCLOSURE}

We would like to thank all the graduate midwives who participated in the survey and provided their views of the programme. This feedback has been hugely valuable. We would also like to thank all of the midwives and mentors who have worked alongside these graduates to provide the holistic support identified by this research. Some funding and research time was provided by Otago Polytechnic and New Zealand College of Midwives.

\section{REFERENCES}

Altier, M., \& Krsek, C. A. (2006). Effects of a 1-year residency program on job satisfaction and retention of new graduate nurses. Journal for Nurses in Staff Development, 22(2), 70-77.

Banks, P., Roxburgh, M., Kane, H., Lauder, W., Jones, M., Kydd, A., \& Atkinson, J. (2011). Flying Start NHS: easing the transition from student to registered health professional. Journal of Clinical Nursing, 20, 3567-3576.

Bolden, L., Cuevas, N., Raia, L., Meredith, E., \& Prince, T. (2011). The use of reflective practice in new graduate registered nurses residency program. Nursing Administration Quarterly, 35(2), 134-139.

Calvert, I. (2001). Midwives should nurture their young, not eat them. New Zealand College of Midwives Journal, 23, 28-29.

Cubit, K. A., \& Ryan, B. (2011). Tailoring a graduate nurse program to meet the needs of our next generation nurses. Nurse Education Today, 31(1), 65-71.

Davis, D., Foureur, M., Clements, V., Brodie, P., \& Herbison, P. (2011). The self-reported confidence of newly graduated midwives before and after their first year of practice in Sydney, Australia. Women and Birth 25(3), e1-e10.

Dixon, L., Calvert, S., Tumilty, E., Kensington, M., Gray, E., Campbell, N., . . Pairman, S. (2015). Supporting New Zealand graduate midwives to stay in the profession: An evaluation of the Midwifery First Year of Practice programme. Midwifery, In press. doi: http://dx.doi. org/10.1016.j.mid.2015.02.010

Dixon, L., Tumilty, E., Kensington, M., Campbell, N., Lennox, S., Calvert, S., . . . Pairman, S. (2014). Stepping forward into life as a midwife in New Zealand/Aotearoa: An analysis of the Midwifery First Year of Practice programme 2007 to 2010. Christchurch: New Zealand College of Midwives.

Fenwick, J., Hammond, A., Raymond, J., Smith, R., Gray, J., Foureur, M., ... Symon, A. (2012). Surviving, not thriving: A qualitative study of newly qualified midwives' experience of their transition to practice. Journal of Clinical Nursing, 21(13-14), 2054-2063. doi: http://dx.doi. org/10.1111/j.1365-2702.2012.04090.x

Goode, C. J., Lynn, M. R., Krsek, C. A., \& Bednash, G. D. (2009). Nurse residency programs: An essential requirement for nursing. Nursing Economic, 27(3), 142-148.
Guilliland, K., Pairman, S., (2010). The midwifery partnership: a model for practice. (2nd.ed.) Christchurch, N.Z.: New Zealand College of Midwives.

Hastie, C. (1995). Midwives eat their young, don't they? Birth Issues, 4(3), 5-9.

Herdrich, B., \& Lindsay, A. (2006). Nurse Residency Programs. Redesigning the transition into practice. Journal for Nurses in Staff Development, 22(2), 55-62.

Hillman, L., \& Foster, R. R. (2011). The impact of a nursing transitions programme on retention and cost savings. Journal of Nursing Management, 19, 50-56.

Hobbs, J., \& Green, S. (2003). Development of a preceptorship programme. British Journal of Midwifery, 11(6), 372-375.

Hobbs, J. A. (2012). Newly qualified midwives' transition to qualified status and role: Assimilating the 'habitus' or reshaping it? Midwifery, 28(3), 391-399. doi: http://dx.doi.org/10.1016/j.midw.2011.04.007

Holland, D. (2001). Practice wisdom: Mentoring, a personal analysis. New Zealand College of Midwives Journal, 23, 15-18.

Kensington, M. (2006). The faces of mentoring in New Zealand: Realities for the new graduate midwife. New Zealand College of Midwives Journal, 35, 22-27.

Lennox, S., Jutel, A. \& Foureur, M. (2012). The Concerns of Competent Novices during a Mentoring Year. Nursing Research and Practice, Open Access Article ID 812542. doi:10.1155/2012/812542

Lennox, S., \& Foureur, M. (2012). Developmental mentoring: New graduates' confidence grows when their needs shape the relationship. New Zealand College of Midwives Journal, 46, 26-31.

Mason, J., \& Davies, S. (2013). A qualitative evaluation of a preceptorship programme to support newly qualified midwives. $R C M$ midwives: the official journal of the Royal College of Midwives, $11(3), 94-$ 98.

New Zealand College of Midwives (2000). Consensus Statement: Mentoring. Christchurch: New Zealand College of Midwives.

Newhouse, R. P., Hoffman, J. J., Suflita, J., \& Hairston, D. P. (2007). Evaluating an Innovative Program to Improve New Nurse Socialization into the Acute Healthcare Setting. Nursing Administration Quarterly, 31(1), 50-60.

O'Malley Floyd, B., Kretschmann, S., \& Young, H. (2005). Facilitating Role Transition for New Graduate RNs in a Semi-Rural Healthcare Setting. Journal for Nurses in Staff Development, 21(6), 284-290.

Pairman, S., Dixon, L., Tumilty, E., Gray, E., Campbell, N., Calvert, S., \& Kensington, M. (2015). The Midwifery First Year of Practice programme supporting New Zealand midwifery graduates in their transition to practice. In Press.

Skirton, H., Stephen, N., Doris, F., Cooper, M., Avis, M., \& Fraser, D. M. ( 2012). Preparedness of newly qualified midwives to deliver clinical care: An evaluation of pre-registration midwifery education through an analysis of key events. Midwifery, 28(5), e660-e666. doi: 10.1016/j. midw.2011.08.007

Solowiej, K., Upton, P., \& Upton, D. (2010). Supporting the transition from student to practitioner: a scheme to support the development of newly qualified practitioners. International Journal of Therapy and Rehabilitation, 17(9), 494-504.

Sullivan, K., Lock, L., \& Homer, C. S. E. (2011). Factors that contribute to midwives staying in midwifery: A study in one area health service in New South Wales, Australia. Midwifery, 27(3), 331-335. doi: http://dx.doi.org/10.1016/j.midw.2011.01.007

Van der Putten, D. (2008). The lived experience of newly qualified midwives: a qualitative study. British Journal of Midwifery, 16(6), 348358.

\section{Accepted for publication April 2016}

Kensington, M., Campbell, N., Gray, E., Dixon, L., Tumilty, E.,

Pairman, S., Calvert, S., Lennox, S., (2016). New Zealand's Midwifery Profession: Embracing graduate midwives' transition to practice. New Zealand College of Midwives Journal, 52, 20-25.

http://dx.doi.org/10.12784/nzcominl52.2016.3.20-25 